Paulo Reis de CARVALHO ${ }^{1}$ Maria Carolina Gonçalves PITA $^{2}$ Eduardo PIBER-NETO² Regina Mieko Sakata MIRANDOLA ${ }^{2}$

Cássio Xavier de MENDONÇA-JÚNIOR²

Correspondência para: PAULOREIS DE CARVALHO Unidade de Pesquisae Desenvolvimento de Bauru

Agência Paulista de Tecnologia do Agronegócio

Secretaria de Agricultura e Abastecimento Av. Rodrigues Alves, 40-40 17100-000 - Bauru - SP

\title{
Influência da adição de fontes marinhas de carotenóides à dieta de galinhas poedeiras na pigmentação da gema do ovo
}

1 - Unidade de Pesquisa e Desenvolvimento de Bauro da Agência Paulista de Tecnologia do Agronegócio da Secretaria de Agricultura e Abastecimento, Bauru - SP

2 - Departamento de Clínica Médica da Faculdade de Medicina Veterinária

e Zotecnia da Universidade de São Paulo, São Paulo - SP

\section{Resumo}

A presente pesquisa foi realizada a fim de estudar os efeitos da suplementação de duas fontes marinhas de carotenóides em concentrações crescentes na dieta sobre a pigmentação da gema de ovos de galinhas poedeiras. O delineamento experimental foi inteiramente casualizado e utilizou 288 galinhas poedeiras com 32 semanas de idade distribuídas em 12 tratamentos, sendo cada unidade experimental constituída de 3 repetições de 8 aves. As concentrações empregadas de óleo de salmão (Salmo salar) foram de 0,80\%; 1,20\%; $1,60 \% ; 2,00 \%$; e 2,40\% e de alga marinha (Schizochytrium sp) de $0,50 \%$; $0,75 \% ; 1,00 \% ; 1,25 \% ; 1,50 \% ;$ e 1,75\%. A dieta basal foi isocalórica e isoprotéica e constituída à base de milho e soja. Quanto à pigmentação da gema, os valores dos equivalentes em $\beta$-caroteno obtidos para as aves que receberam óleo de salmão na dieta variaram significativamente $(\mathrm{P}<0,05)$ de 27,04 $\mu \mathrm{g} / \mathrm{g}$ a 45,96 $\mu \mathrm{g} / \mathrm{g}$ de gema, respectivamente, para $0,80 \%$ e 2,40\% deste óleo. Para as aves alimentadas com alga, as médias oscilaram de $33,04 \mu \mathrm{g} / \mathrm{g}$ a $72,66 \mu \mathrm{g} / \mathrm{g}$ gema para as dietas apresentando, respectivamente, de $0,50 \%$ a $1,75 \%$ de alga. $\mathrm{O}$ escore visual (leque colorimétrico Roche) das gemas mostrou diferenças significativas $(\mathrm{P}<0,05)$ entre as médias dos tratamentos que oscilaram de 4,75 (0,80\% de óleo de salmão) a 8,17 (2,40\% do mesmo óleo). Para as dietas enriquecidas com alga os valores observados foram de 5,92 a 11,58 , respectivamente, para percentuais de $0,50 \%$ e $1,75 \%$ desta fonte alimentar. O parâmetro de qualidade do albúmen, medido em unidades Haugh, mostrou diferenças significativas $(\mathrm{P}<0,05)$ para as médias dos tratamentos em relação ao grupo controle. Os coeficientes de correlação e de determinação observados entre os percentuais de óleo de salmão ou de alga na dieta e os teores de equivalentes $\beta$-caroteno na gema $(\mu \mathrm{g} / \mathrm{g})$ evidenciaram a existência de linearidade significativa $(\mathrm{P}<0,05)$ entre estas variáveis.

\section{Introdução}

A importância fundamental dos carotenóides na nutrição de aves reside no fato de serem estas substâncias os principais grupos de compostos responsáveis pela coloração da gema do ovo e da pele do frango, podendo ser controlados pela
Palavras-chave:

Pigmentação da gema.

Poedeiras.

Carotenóides.

Fontes marinhas. $\operatorname{dieta}^{1,2}$. Os carotenóides são fitonutrientes que se apresentam na natureza com cores oscilando do amarelo ao vermelho, passando pelo laranja, variação esta resultante das duplas ligações conjugadas e alternadas com ligações simples na estrutura carbônica. Assim, o $\beta$-caroteno com onze duplas ligações conjugadas que formam o sistema 
cromóforo tem maior absorção na região do espectro visível com comprimento de onda máximo entre 420 a $510 \mathrm{~nm}$, sendo verde-amarelo na aparência ${ }^{3}$. O $\beta$-caroteno, zeaxantina, luteína, astaxantina, cantaxantina e criptoxantina fazem parte da família dos carotenóides e apresentam formula estrutural e funções bioquímicas semelhantes. Os carotenóides, quando presentes na alimentação, têm sido associados à redução do risco de câncer, catarata, degeneração macular senil e imunodeficiência ${ }^{4,5,6,7,8,9,10}$. Tais compostos participam ainda de funções vitais, tanto no homem como nos animais, fazendo parte de pigmentos estruturais importantes ${ }^{11,12}$. Neste sentido, a mácula da retina apresenta a luteína, zeaxantina, mesozeaxantina que têm a função de absorver a luz azul protegendo as células fotoreceptoras, dos cones e bastonetes, de danos conseqüentes da radiação eletromagnética de comprimento de onda no espectro visível, durante a reação fotossensitiva ${ }^{7,13}$.

Vários compostos de fórmulas estruturais isoméricas ou derivados de carotenóides têm a capacidade de serem convertidos em vitamina A. Leeson e Summers ${ }^{14}$, descrevem que em aves esta conversão é estimada em 3 a 4 moles de $\beta$-caroteno para $1 \mu \mathrm{g}$ de vitamina $A$. Ben-Amotz, Edelstein e Avron ${ }^{15}$ verificaram que a adição de um grama da alga Dunaliella bardawil por quilo de dieta de pintos correspondia a um suprimento de aproximadamente $30 \mathrm{mg}$ de $\beta$-caroteno $/ \mathrm{kg}$. Hudon $^{16}$ descreveu diferença no teor de astaxantina ${ }^{17}$ existente no salmão do atlântico e no salmão criado em tanques, sendo que estes apresentaram maiores concentrações de xantofilas amarelas. Herber-Mcneill e Van Elswkyk $^{18}$ estudaram o efeito da adição $2,4 \%$ e $4,8 \%$ da alga marinha Schizochytrium na alimentação de poedeiras sobre a pigmentação da gema de ovos e como resposta obtiveram diferença significativa a nível de 1\% no aumento da coloração da gema. Silva, Albino e Godói ${ }^{19}$ avaliaram a adição de níveis crescentes de extrato de urucum de 0,$10 ; 0,15 ; 0,30 ; 0,45 ;$ e $0,60 \%$ adicionados à ração de duas linhagens de galinhas poedeiras e observaram resultados com efeito linear positivo para a pigmentação da gema em função do aumento do percentual deste corante $^{20}$ na alimentação. Schrenk, Chapin e Conrad ${ }^{21}$ citados por Marusich e Bauernfeind ${ }^{22}$ relataram que os carotenóides são depositados na gema do ovo em proporção direta à sua concentração na ração, sendo que alguns podem ser depositados em outros tecidos ou não absorvidos.

Entre os principais pré-requisitos que determinam a preferência do consumidor por produtos alimentícios, relacionada à aparência, destaca-se a cor. Uma dieta formulada com alimentos naturais ricos em carotenóides pode determinar a intensidade da pigmentação da gema de ovos de galinhas poedeiras obtendo-se diferentes graus de intensidade de cor variando do claro despigmentado na dieta à base de milho branco, ao amarelo intenso ou laranja com tonalidade avermelhada nas dietas basais de milho das variedades com altas concentrações destes pigmentos ${ }^{23} \mathrm{ou}$, alternativamente, com fontes suplementares ricas em compostos carotenóides ${ }^{24}$ como algas marinhas ${ }^{25}$ ou alfafa ${ }^{26,27}$ apresentando valores de carotenóides variando de $22 \mu \mathrm{g} / \mathrm{g}$ no milho, $450 \mu \mathrm{g} / \mathrm{g}$ na alfafa desidratada e $1450 \mu \mathrm{g} / \mathrm{g}$ no extrato seco de alga.

Madiedo, Richter e Sunde ${ }^{25}$, Bartov e Bornstein $^{26,28}$ e Bornstein e Bartov ${ }^{1}$ pesquisaram varias dietas para analisar a influência de diferentes níveis de suplementos enriquecidos com altos teores de carotenóides de fontes naturais e sua relação quantitativa com o transporte para a gema do ovo de galinhas poedeiras. Os autores, utilizando rações à base de alga e alfafa, conceituaram equivalente $\beta$-caroteno como sendo a mistura quantitativa de carotenóides presentes na gema provenientes das diferentes dietas experimentais ricas em carotenóides pigmentantes, possíveis de serem detectados e medidos por espectrofotometria no espectro vísivel entre 420 e $510 \mathrm{~nm}$, apresentando cores variando 
do amarelo ao laranja e vermelho quando solubilizados. Fletcher et al. ${ }^{29}$ pesquisaram varias dietas ricas em compostos carotenóides e verificaram a linearidade da concentração da dieta com os teores de equivalentes $\beta$-caroteno na gema de ovos de poedeiras à partir do décimo segundo dia do início do experimento.

O objetivo da presente pesquisa é de quantificar a pigmentação da gema do ovo em equivalentes $\beta$-caroteno, mediante emprego de espectrofotometria de luz visível, e em escore visual, com valores obtidos pelo leque colorimétrico Roche, em aves submetidas a teores crescentes de óleo de salmão ou alga marinha acrescidos a dieta basal de milho e soja.

\section{Materiais e Métodos}

A pesquisa foi conduzida de outubro a dezembro de 2003 no biotério experimental de aves do Departamento de Clínica Médica da Faculdade de Medicina Veterinária e Zootecnia da Universidade de São Paulo.

No presente estudo foi utilizado delineamento inteiramente casualizado, empregando-se 288 galinhas poedeiras da linhagem comercial Hisex White com 32 semanas idade, distribuídas em 12 tratamentos, com três repetições de oito aves, alojadas em gaiolas de $0,45 \mathrm{~m} \times 0,25 \mathrm{~m}$ x $0,45 \mathrm{~m}$, sendo duas aves por gaiola. A ração foi fornecida ad libitum em comedouro tipo calha e a água em bebedouro tipo nipple. As aves receberam 16 horas diárias de luz.

As rações experimentais, isocalóricas e isoproteicas, foram formuladas de acordo com os requerimentos estabelecidos pelo $\mathrm{NRC}^{30}$. O grupo controle foi composto de dieta basal de milho e soja, enquanto que, nos demais tratamentos, as rações foram suplementadas com óleo de salmão ou alga. Os tratamentos de dois a seis tiveram adição de proporções crescentes de óleo de salmão (Salmo salar) nas concentrações de $0,80 \%$; $1,20 \% ; 1,60 \% ; 2,00 \%$; e 2,40\%, enquanto que os de sete a doze continham percentuais crescentes de alga marinha (Schizochytrium sp) de $0,50 \% ; 0,75 \% ; 1,00 \% ; 1,25 \% ; 1,50 \%$ e 1,75\% (Tabela 1). Ao óleo de salmão e alga marinha foram adicionados $200 \mathrm{ppm}$ do antioxidante butil hidroxi tolueno.

Os ovos utilizados para a determinação da pigmentação foram coletados na oitava semana experimental, sendo o escore visual obtido de um pool de quatro gemas, por repetição, sendo avaliado por quatro analistas, em ambiente iluminado e confrontado à escala de 1 a 15 do leque colorimétrico $^{31}$.

A quantificação dos carotenóides presentes na gema foi realizada por espectrofotometria de luz visível utilizando o comprimento de onda de $445 \mathrm{~nm}$, de acordo com técnica descrita pela $\mathrm{AOAC}^{32}$ para determinação de coloração de gemas de ovos em equivalentes $\beta$-caroteno, após calibração do aparelho utilizando padrão de $\beta$-caroteno Hoffman La Roche. Foi utilizada a fórmula expressa pela lei de LambertBeer, citada por Skoog, Holler e Nieman ${ }^{3}$.

Para avaliação da qualidade do albúmen $^{33}$, foram utilizados doze ovos por tratamento, empregando-se micrômetro Ames S- $8400^{\circledR}$.

A análise estatística dos dados experimentais foi realizada utilizando-se o software $\mathrm{SAS}^{34}$, sendo as comparações entre as médias dos tratamentos efetuadas mediante teste de Tukey, adotando-se o nível de $5 \%$ de significância.

\section{Resultados e Discussão}

Os pesos médios do ovo e da gema não apresentaram diferenças significativas pela adição crescente de óleo de salmão à dieta, na $8^{a}$ semana experimental (Tabela 2).

Os valores de equivalentes $\beta$-caroteno, expressos tanto por grama como pelo total da gema, dos ovos das aves submetidas a dietas contendo diferentes percentuais de óleo de salmão, variaram de forma linear de 24,10 a 45,96 $\mu \mathrm{g} / \mathrm{g}$ de gema e de 400,09 a $754,46 \mu \mathrm{g} /$ gema total, sendo as diferenças entre médias consideradas estatísticamente 
Tabela 1 - Composição da ração basal. São Paulo, 2003

\begin{tabular}{|c|c|}
\hline Ingrediente & $\%$ \\
\hline Milho & 53,79 \\
\hline Farelo de soja & 28,30 \\
\hline Farelinho de trigo & 3,17 \\
\hline Calcário & 9,60 \\
\hline Óleo de milho & 3,00 \\
\hline Fosfato bicálcico & 1,30 \\
\hline Sal comum & 0,30 \\
\hline DL-metionina & 0,27 \\
\hline Cloreto de colina & 0,12 \\
\hline Premix vitamínico ${ }^{1}$ & 0,10 \\
\hline Premix mineral $^{2}$ & 0,05 \\
\hline \multicolumn{2}{|l|}{ Análise Calculada } \\
\hline Proteína bruta (\%) & 17 \\
\hline Energia metabolizável (kcal/kg) & 2800 \\
\hline Cálcio (\%) & 4,00 \\
\hline Fósforo disponível (\%) & 0,37 \\
\hline Lisina (\%) & 0,92 \\
\hline Metionina + cistina $(\%)$ & 0,84 \\
\hline
\end{tabular}

${ }^{1}$ Premix vitamínico (composição/ kg): vit. A 8.000.000 U.l; vit. D 2.500.000 U.l; vit. E 10.000 U.l; vit. $K_{3} 2,5$ g; vit. $B_{1} 1$ g; vit. $B_{2} 5$ g; vit. ${ }_{6} 1,5$ g; vit. $\mathrm{B}_{12} 12 \mathrm{mg}$; ácido nitotínico $25 \mathrm{~g}$; ácido pantotênico 8 g; ácido fólico 500 mg; selênio $150 \mathrm{mg}$.

${ }^{2}$ Premix mineral (composição/ kg): ferro 100 g; cobre 20 g; cobalto 2 g; manganês 160 g; zinco 100 g; iodo 2 g.

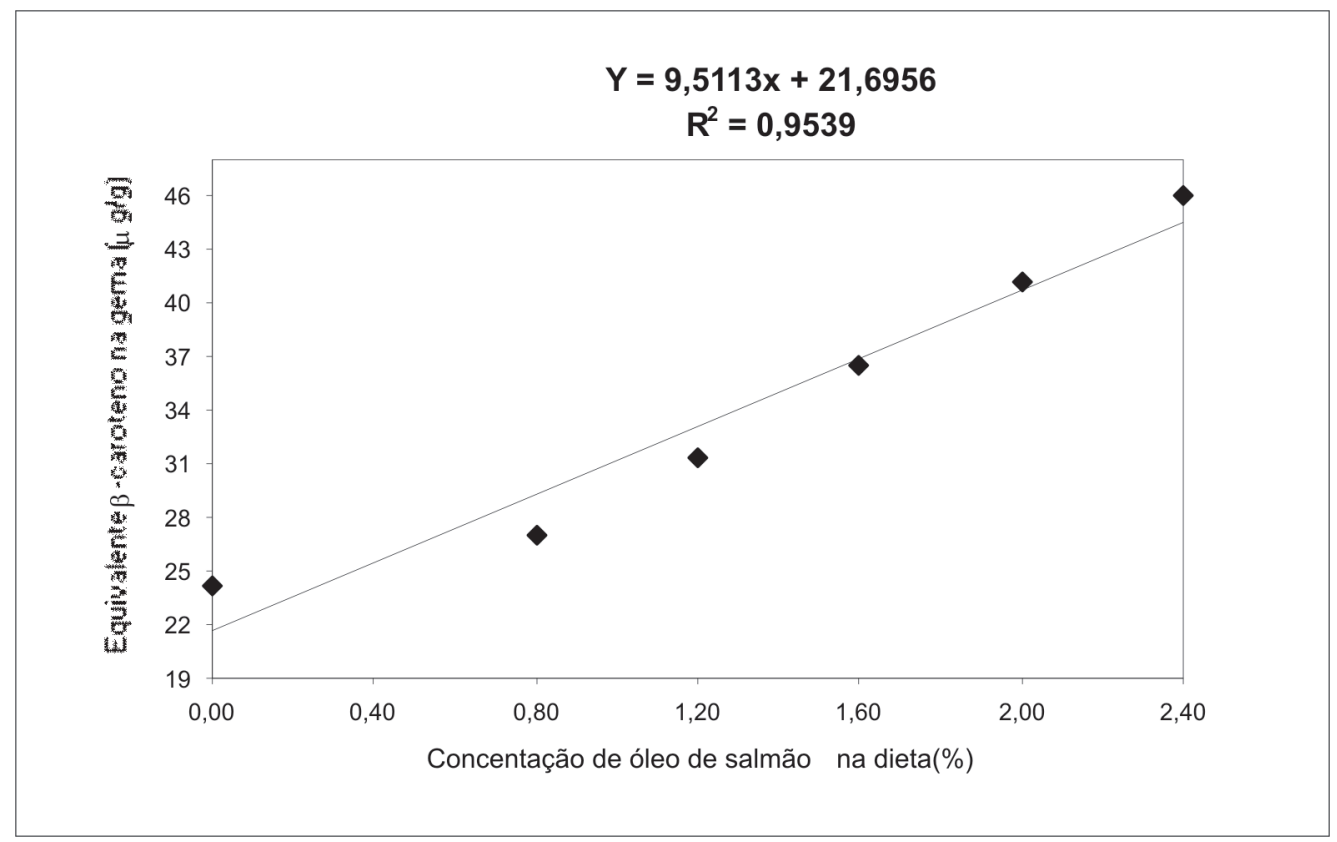

Figura 1-Equivalente $\beta$-caroteno ( $\mu \mathrm{g} / \mathrm{g}$ ) na gema em função concentração de óleo de salmão na dieta 
Tabela 2-Peso do ovo, peso, equivalente $\beta$-caroteno e escore visual da gema, qualidade do albúmen e respectivos desvios padrões, segundo os percentuais de óleo de salmão utilizados na dieta. São Paulo, 2003

\begin{tabular}{|c|c|c|c|c|c|c|}
\hline $\begin{array}{c}\text { Óleo de } \\
\text { salmão } \\
\text { na Dieta } \\
(\%)\end{array}$ & $\begin{array}{l}\text { Peso do } \\
\text { ovo } \\
\text { (g) }\end{array}$ & $\begin{array}{l}\text { Peso da } \\
\text { Gema } \\
\text { (g) }\end{array}$ & $\begin{array}{c}\text { Equiv. } \beta- \\
\text { caroteno } \\
\text { na gema } \\
(\mu \mathrm{g} / \mathrm{g})\end{array}$ & $\begin{array}{c}\text { Equiv. } \beta \text { - } \\
\text { caroteno } \\
\text { na gema } \\
\text { ( } \mu \text { g/gema) }\end{array}$ & $\begin{array}{l}\text { Escore } \\
\text { visual }\end{array}$ & $\begin{array}{c}\text { Unidades } \\
\text { Haugh } \\
(\%)\end{array}$ \\
\hline 0,00 & $63,25^{\mathrm{a}}$ & $16,60^{a}$ & $24,10^{a}$ & $\begin{array}{c}400,09^{\mathrm{a}} \\
+17,19\end{array}$ & $\begin{array}{l}3,75^{\mathrm{a}} \\
+045\end{array}$ & $89,03^{a}$ \\
\hline 0,80 & $\begin{array}{r}63,96^{\mathrm{a}} \\
+4,37\end{array}$ & $\begin{array}{r}16,43^{a} \\
\pm 1,17\end{array}$ & $\begin{array}{c}27,04^{\mathbf{b}} \\
\pm 0,12\end{array}$ & $\begin{array}{c}444,30^{\mathrm{b}} \\
\pm 4,79\end{array}$ & $\begin{array}{l}4,75^{\mathbf{b}} \\
+0,62\end{array}$ & $\begin{array}{c}12,00 \\
88,78^{\text {a }} \\
\pm 1,97\end{array}$ \\
\hline 1,20 & $\begin{array}{l}66,30^{\mathrm{a}} \\
\pm 7,07\end{array}$ & $\begin{array}{r}16,91^{\mathrm{a}} \\
\pm 1,43\end{array}$ & $\begin{array}{c}31,37^{\mathrm{c}} \\
\pm 0,20\end{array}$ & $\begin{array}{r}530,81^{\mathrm{c}} \\
\pm 12,57\end{array}$ & $\begin{array}{l}5,25^{\mathrm{bc}} \\
\pm 0,62\end{array}$ & $\begin{array}{c}88,61^{\text {a }} \\
\pm 2,23\end{array}$ \\
\hline 1,60 & $\begin{array}{l}62,08^{a} \\
\pm 3,37\end{array}$ & $\begin{array}{r}16,06^{a} \\
\pm 1,16\end{array}$ & $\begin{array}{l}36,58^{\mathrm{d}} \\
\pm 0,19\end{array}$ & $\begin{array}{r}587,52^{\mathrm{d}} \\
\pm 20,70\end{array}$ & $\begin{array}{r}5,75^{\mathrm{c}} \\
\pm 0,75\end{array}$ & $\begin{array}{c}88,56^{\text {a }} \\
\pm 2,42\end{array}$ \\
\hline 2,00 & $\begin{array}{l}61,06^{\mathrm{a}} \\
\pm 3,45\end{array}$ & $\begin{array}{r}15,74^{a} \\
\pm 1,07\end{array}$ & $\begin{array}{l}41,20^{\mathrm{e}} \\
\pm 0,04\end{array}$ & $\begin{array}{r}648,90^{\mathrm{e}} \\
\pm 16,44\end{array}$ & $\begin{array}{l}6,58^{d} \\
\pm 0,51\end{array}$ & $\begin{array}{c}88,28^{\text {a }} \\
\pm 1,70\end{array}$ \\
\hline 2,40 & $\begin{array}{l}63,48^{\mathrm{a}} \\
\pm 3,94\end{array}$ & $\begin{array}{r}16,41^{\mathrm{a}} \\
\pm 0,98\end{array}$ & $\begin{array}{l}45,96^{f} \\
\pm 0,90\end{array}$ & $\begin{array}{r}754,46^{\mathrm{f}} \\
\pm 27,82\end{array}$ & $\begin{array}{l}8,17^{\mathbf{e}} \\
\pm 1,02\end{array}$ & $\begin{array}{c}88,22^{\text {a }} \\
\pm 2,99\end{array}$ \\
\hline
\end{tabular}

Médias na coluna com letras distintas diferem $(\mathrm{P}<0,05)$ entre si pelo teste de Tukey

significativas (Tabela 2). De fato, os coeficientes de correlação $(r=0,9768)$ e de determinação $\left(r^{2}=0,9539\right)$, assinalados entre os teores de óleo de salmão na dieta e os equivalentes $\beta$-caroteno na gema $(\mu \mathrm{g} / \mathrm{g})$ mostraram-se de significância estatística (Figura 1). Tais resultados estão de acordo com Bartov e Bornstein ${ }^{26,28}$, Bornstein e Bartov $^{1}$ e Madiedo, Richter e Sunde ${ }^{25}$ que relataram valores para equivalentes $\beta$-caroteno superiores a $30 \mu \mathrm{g} / \mathrm{g}$ de gema para aves submetidas a dietas com carotenóides, evidenciando linearidade entre as concentrações de carotenóides presentes na dieta e equivalentes $\beta$-caroteno da gema. Marusich e Bauernfeind ${ }^{22}$, por sua vez, ressaltaram que os carotenóides são transferidos para o ovo 48 horas após a ingestão alimentar, sendo que sua concentração na gema está na razão direta do teor de carotenóides presente na ração.

A amplitude dos valores do escore visual das gemas obtidos entre os tratamentos utilizando diferentes teores de óleo de salmão foi de 3,75 a 8,17 evidenciando linearidade entre tais variáveis $\left(r=0,8844\right.$ e r $\left.^{2}=0,7821\right)$ (Tabela 2 e Figura 3). Tais resultados estão de acordo com Café et al. ${ }^{35}$ que assinalaram valores médios de cor da gema pelo escore do leque colorimétrico de 7,28; 6,22; 4,90; 3,$55 ; 1,30$ utilizando milheto em níveis crescentes de, respectivamente, $0 ; 25 ; 50 ; 75$; e $100 \%$ em substituição ao milho da dieta de galinhas poedeiras, evidenciando regressão linear com $\mathrm{r}^{2}=0,9841$.

Quando foi utilizado o óleo de salmão na dieta, valores médios da qualidade interna do ovo, obtidos em unidades Haugh, não apresentaram diferenças significativas em relação ao grupo controle (Tabela 2). Estes dados estão de acordo com Herber e Van Elswyk ${ }^{36}$ que avaliando a influência da adição de 1,5\% óleo de menhaden, e de $2,4 \%$ e $4,8 \%$ de alga marinha à dieta de poedeiras, não observaram alteração nos parâmetros de qualidade dos ovos, mas obtiveram melhora significativa na pigmentação da gema. No padrão americano de classificação de ovos, segundo Oliveira $^{37}$, ovos com unidades Haugh de $72 \%$ são considerados de excelente qualidade. No presente estudo, o menor valor de qualidade de albúmen foi de $88,22 \%$ obtido para aves alimentadas com dieta contendo $2,4 \%$ de óleo de salmão.

A adição de alga marinha à dieta das poedeiras não determinou diferenças significativas tanto no peso médio do ovo 
Tabela 3 - Peso do ovo, peso, equivalente $\beta$-caroteno e escore visual da gema, qualidade do albúmen e respectivos desvios padrões, segundo os percentuais de alga marinha utilizados na dieta. São Paulo, 2003

\begin{tabular}{|c|c|c|c|c|c|c|}
\hline $\begin{array}{c}\text { Alga } \\
\text { marinha } \\
\text { dieta } \\
(\%)\end{array}$ & $\begin{array}{c}\text { Peso } \\
\text { ovo } \\
(g)\end{array}$ & $\begin{array}{c}\text { Peso } \\
\text { Gema } \\
(\mathrm{g})\end{array}$ & $\begin{array}{c}\text { Equiv. } \beta \text { - } \\
\text { caroteno } \\
\text { na gema } \\
(\mu \mathrm{g} / \mathrm{g})\end{array}$ & $\begin{array}{l}\text { Equiv. } \beta \text { - } \\
\text { caroteno } \\
\text { na gema } \\
\text { ( } \mu \text { g/gema) }\end{array}$ & $\begin{array}{l}\text { Escore } \\
\text { Visual }\end{array}$ & $\begin{array}{c}\text { Unidade } \\
\text { Haugh } \\
(\%)\end{array}$ \\
\hline 0,00 & $\begin{array}{r}63,25^{\mathrm{a}} \\
\pm 2,35\end{array}$ & $\begin{array}{r}16,60^{a} \\
\pm 1,16\end{array}$ & $\begin{array}{l}24,10^{a} \\
\pm 0,03\end{array}$ & $\begin{array}{r}400,09^{a} \\
\pm 17,19\end{array}$ & $\begin{array}{l}3,75^{\mathrm{a}} \\
\pm 0,45\end{array}$ & $\begin{array}{c}89,030^{\mathrm{a}} \\
\pm 2,68\end{array}$ \\
\hline 0,50 & $\begin{array}{c}62,96^{\mathrm{a}} \\
\pm 4,54\end{array}$ & $\begin{array}{r}17,07^{a} \\
\pm 1,34\end{array}$ & $\begin{array}{l}33,04^{\mathrm{a}} \\
\pm 0,14\end{array}$ & $\begin{aligned} & 564,29^{a} \\
\pm & 18,68\end{aligned}$ & $\begin{array}{l}5,92^{\mathrm{a}} \\
\pm 0,79\end{array}$ & $\begin{array}{c}90,64 \text { b } \\
\pm 2,32\end{array}$ \\
\hline 0,75 & $\begin{array}{c}65,90^{a} \\
\pm 5,15\end{array}$ & $\begin{array}{r}17,18^{a} \\
\pm 0,97\end{array}$ & $\begin{array}{l}39,81^{\mathrm{b}} \\
\pm 0,08\end{array}$ & $\begin{aligned} & 684,22^{b} \\
\pm & 27,41\end{aligned}$ & $\begin{array}{l}7,08^{b} \\
\pm 0,66\end{array}$ & $\begin{array}{c}90,67 \mathrm{~b} \\
\pm 1,67\end{array}$ \\
\hline 1,00 & $\begin{array}{c}63,40^{\mathrm{a}} \\
\pm 3,45\end{array}$ & $\begin{array}{r}16,28^{a} \\
\pm 1,07\end{array}$ & $\begin{array}{l}48,62^{c} \\
\pm 0,15\end{array}$ & $\begin{array}{c}791,59^{\mathrm{c}} \\
\pm 48,27\end{array}$ & $\begin{array}{l}8,42^{c} \\
\pm 0,90\end{array}$ & $\begin{array}{c}90,78 \text { bc } \\
\pm 1,76\end{array}$ \\
\hline 1,25 & $\begin{array}{c}64,20^{a} \\
\pm 4,23\end{array}$ & $\begin{array}{r}16,00^{a} \\
\pm 2,16\end{array}$ & $\begin{array}{l}56,67^{d} \\
\pm 0,13\end{array}$ & $\begin{array}{r}907,04^{d} \\
\pm 35,43\end{array}$ & $\begin{array}{l}9,50^{d} \\
\pm 0,67\end{array}$ & $\begin{array}{c}91,11 \text { bc } \\
\pm 2,00\end{array}$ \\
\hline 1,50 & $\begin{array}{l}65,15^{a} \\
\pm 2,68\end{array}$ & $\begin{array}{r}17,00^{\mathrm{a}} \\
\pm 0,64\end{array}$ & $\begin{array}{l}64,08^{\mathrm{e}} \\
\pm 0,04\end{array}$ & $\begin{array}{r}1089,62^{\mathrm{e}} \\
\pm 20,83\end{array}$ & $\begin{array}{c}10,75^{\mathrm{e}} \\
\pm 0,75\end{array}$ & $\begin{array}{c}91,28 \text { bc } \\
\pm 1,75\end{array}$ \\
\hline 1,75 & $\begin{array}{c}65,28^{a} \\
\pm 5,08\end{array}$ & $\begin{array}{r}16,38^{a} \\
\pm 1,12 \\
\end{array}$ & $\begin{array}{r}72,66^{f} \\
\pm 0,20\end{array}$ & $\begin{array}{r}1190,41^{f} \\
\pm 38,11 \\
\end{array}$ & $\begin{array}{c}11,58^{\mathrm{e}} \\
\pm 0,66\end{array}$ & $\begin{array}{c}92,17 \mathrm{c} \\
\pm 2,04\end{array}$ \\
\hline
\end{tabular}

Médias na coluna com letras distintas diferem $(\mathrm{P}<0,05)$ entre si pelo teste de Tukey

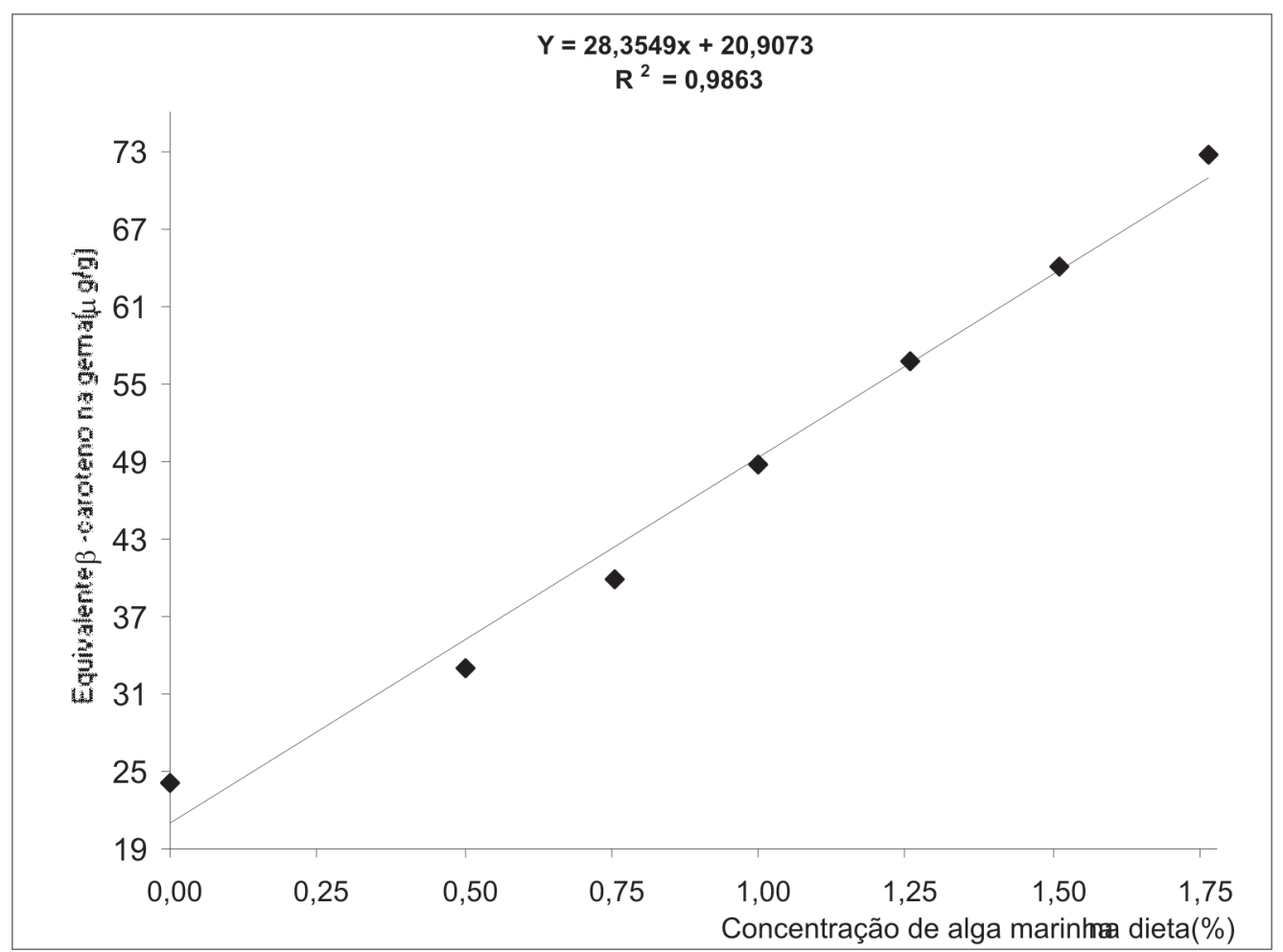

Figura 2-Equivalente $\beta$-caroteno $(\mu \mathrm{g} / \mathrm{g})$ na gema em função concentração de alga marinha na dieta 


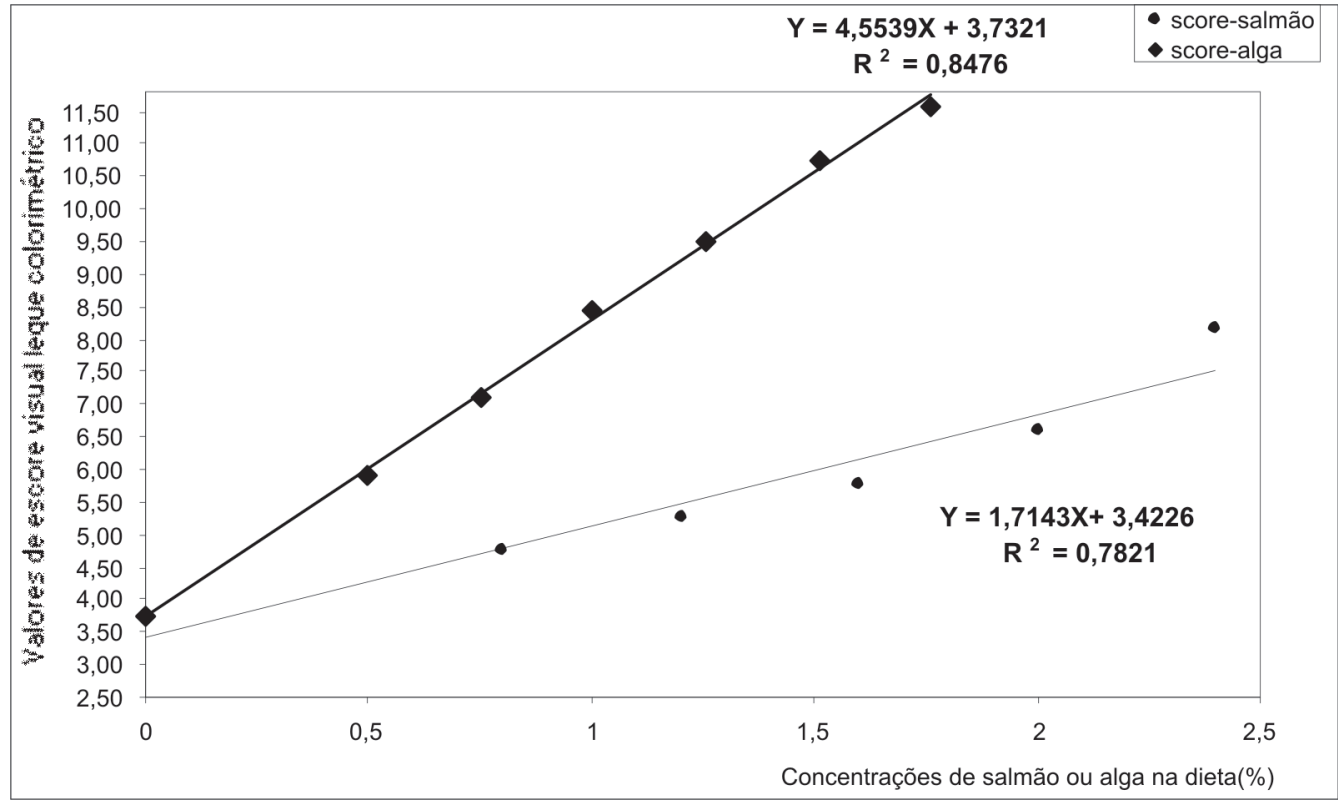

Figura 3 - Escore visual Color Fan Roche em função das fontes óleo de salmão e alga marinha na dieta

como no da gema, às 8 semanas experimentais (Tabela 3).

Quanto à relação entre os níveis crescentes de alga marinha adicionados à dieta basal, foi assinalada linearidade entre os percentuais na dieta e valores de equivalentes $\beta$-caroteno na gema. Os dados evidenciaram elevados e significativos coeficientes de correlação ( $\mathrm{r}=0,9931)$ e de determinação $\left(r^{2}=0,9863\right)$ entre teores de alga marinha alimentar e equivalentes $\beta$-caroteno da gema $(\mu \mathrm{g} / \mathrm{g})$ (Figura 2). Considerando a variação observada nos valores destes equivalentes 24,10 a 72,66 $\mu \mathrm{g} / \mathrm{g}$ gema e 400,09 a 1190,41 $\mu \mathrm{g} /$ gema total (Tabela 3) - observa-se aumento expressivo, da ordem de 300\%, nestes teores. As diferenças significativas para equivalente $\beta$-caroteno na gema em relação as dietas de óleo de salmão e alga nos tratamentos, foram mais acentuadas para esta última em relação a primeira (Tabela 3).

A qualidade interna dos ovos, ao serem utilizados níveis de alga entre 0,50\% $(90,64 \%)$ e $1,75 \%(92,17 \%)$ na dieta, apresentou diferenças significativas em relação ao controle (89,03\%), sendo evidente a melhora progressiva da qualidade pela elevação dos percentuais de alga na dieta das aves. Estes dados estão de acordo com Herber-Mcneill e Van Elswyk ${ }^{18}$ e Herber e Van Elswyk ${ }^{36}$ que observaram melhora significativa na cor da gema, aceitabilidade e parâmetros de qualidade dos ovos ao utilizarem níveis de $2,4 \%$ ou 4,8 \% de alga marinha na ração de poedeiras.

As concentrações de equivalentes $\beta$-caroteno na gema do ovo das aves que receberam alga marinha na dieta variaram significativamente de 24,10 a 72,66 mg/g de gema correspondendo a uma leitura de escore visual na escala de 3,75 a 11,58, apresentando significativos coeficientes de correlação $(r=0,9206)$ e determinação $\left(\mathrm{r}^{2}=0,8476\right)$ (Tabela 3 e Figura 3). As médias de escore na fonte alga marinha diferiram significativamente em todas as combinações, com exceção das comparações entre $0 \%$ $(3,75)$ e $0,5 \%(5,92)$ e entre $1,50 \%(10,75)$ e $1,75 \%(11,58)$ que não diferiram entre si (Tabela 3). Citações da mesma natureza são encontradas no estudo realizado por Silva, Albino e Godói ${ }^{19}$, que avaliaram coloração da gema pelo leque colorimétrico em duas linhagens comerciais de poedeiras, Lohmann e Isa Brown, com inclusão de níveis crescentes de extrato oleoso de urucum na 
dieta, obtendo efeitos lineares para a pigmentação da gema com valores de $\mathrm{r}^{2} \mathrm{de}$ 0,98 e 0,99 , respectivamente.

Os dados da tabela 3 confirmam a superioridade pigmentante da alga marinha em relação ao óleo de salmão conforme descrito por Madiedo, Richter e Sunde ${ }^{25}$, Schofield, Evens e Millie ${ }^{38}$.

Sendo a alga um fitonutriente de substrato vegetal seco, com concentrações mais elevadas em carotenóides ${ }^{22,30,36}$ e com tendência a pigmentar intensamente na cor amarela, esta apresentou diferenças acentuadas em relação ao óleo de salmão (Tabelas 2 e 3, Figura 3) que revelou tendência para pigmentar levemente na cor de transição entre o amarelo e o laranja, expressando na interpretação da cor visual como sendo de tonalidade alaranjada ${ }^{17,39,40}$. Para avaliações desta natureza em ingredientes semelhantes ao óleo de salmão, Fletcher ${ }^{41,42}$ sugeriu leituras no colorímetro de triestimulo que avalia cor, tonalidade e brilho. Entretanto, vários autores ${ }^{1,25,43}$ consideram a quantificação dos substratos dos ingredientes e da gema o método mais adequado para avaliar a pigmentação da gema do ovo em termos de equivalentes $\beta$-caroteno em $\mu \mathrm{g} / \mathrm{g}$ gema $^{22,25,26,44}$, o que também foi verificado no presente trabalho, no qual foram utilizados dois métodos de avaliação, ou seja, a quantificação em equivalentes $\beta$-caroteno e o escore visual.

As dietas experimentais proporcionaram pigmentação das gemas com carotenóides naturais em valores crescentes pela avaliação do escore visual, transferindo segurança ao observador de que os ovos apresentavamse enriquecidos quando comparados ao parâmetro controle. Os níveis extremos de ambos os ingredientes, óleo de salmão ou alga, proporcionaram as melhores projeções visuais de pigmentação, aspecto este que também beneficia a saúde, desperta o interesse e determina a preferência do consumidor.

\section{Conclusões}

O presente trabalho permite concluir que os carotenóides provenientes do óleo de salmão ou de alga, presentes nas dietas experimentais em níveis crescentes, foram transferidos linearmente para o ovo, resultando em diferenças significativas na pigmentação da gema entre os tratamentos estudados e avaliados tanto pelo leque colorimétrico (escore visual) como pelos equivalentes $\beta$-caroteno.

A correlação linear consignada entre teores das fontes de carotenóides da dieta e pigmentação da gema, evidenciou valores elevados e significativos. $O$ prolongamento do período experimental em futuras pesquisas certamente permitirá chegar a outras conclusões mais aprofundadas em relação a relevância do papel bioquímico e a importância dos carotenóides como nutrientes essenciais para galinhas de postura.

Os ovos em todos os tratamentos estudados apresentaram-se de ótima qualidade interna, com valores de unidades Haugh acima de $88,22 \%$. A adição de alga à dieta, entre $0,50 \%$ e $1,75 \%$, proporcionou progressiva e significativa melhora na qualidade interna dos ovos e na pigmentação da gema avaliada pelos equivalentes $\beta$-caroteno e escore visual.

\section{Influence of sea sources of carotenoids added to the diet of laying hens on the egg yolk pigmentation}

\section{Abstract}

This experiment was carried out to evaluate the effect of the utilization of two sources of marine carotenoids added to the diet on the egg yolk pigmentation of laying hens. A completely randomized design using 288 laying hens with 32 weeks of age assigned in 12 treatments, with 3 repetitions of 8 layers each was used. The hens were fed a corn
Key-words: Egg yolk.

Pigmentation. Laying hens.

Carotenoids.

Marine sources. 
soybean basal diet supplemented with $0.80 \% ; 1.20 \% ; 1.60 \% ; 2.00 \%$; $2.40 \%$ salmon oil (Salmo salar) or $0.50 \% ; 0.75 \% ; 1.00 \% ; 1.25 \%$; $1.50 \%$; and $1.75 \%$ of marine algae (Schizochytrium sp). The diets were isocaloric and isoproteic. The yolk values of $\beta$-carotene equivalents $(\mu \mathrm{g} / \mathrm{g}$ egg yolk) for the laying hens fed salmon oil diet ranged significantly $(\mathrm{P}<0.05)$ from $27.04 \mu \mathrm{g} / \mathrm{g}$ to $45.96 \mu \mathrm{g} / \mathrm{g}$, respectively, for $0.80 \%$ and $2.40 \%$ salmon oil. Laying hens fed marine algae showed $\beta$-carotene equivalent means ranging from $33.04 \mu \mathrm{g} / \mathrm{g}$ to $72.66 \mu \mathrm{g} / \mathrm{g}$ egg yolk, respectively, for $0.50 \%$ and $1.75 \%$ of marine algae diets. Visual score for the egg yolk pigmentation at the eighth week of experiment showed significance $(\mathrm{P}<0.05)$ among means ranging from 4.75 to 8.17 for $0.80 \%$ and $2.40 \%$ salmon oil diets, respectively. The yolk pigmentation measured by visual score (colorimetric fan) of eggs from hens fed diets enriched with marine algae ranged from $5.92(0.50 \%$ algae $)$ to $11.58(1.75 \%$ algae $)$. Haugh units were significantly $(\mathrm{P}<0.05)$ improved when marine algae was added to the basal diet. The significant $(\mathrm{P}<0.05)$ correlation and determination coefficients observed between the percentages of salmon oil or marine algae in the diet and the $\beta$-carotene equivalents found into the yolk $(\mu \mathrm{g} / \mathrm{g})$ showed a strong positive linearity between these variables.

\section{Referências}

1 BORNSTEIN, S.; BARTOV, I. Studies on egg yolk pigmentation. I. A comparison between visual scoring of yolk color and colorimetric assay of yolk carotenoids. Poult. Sci., v. 45, n. 2, p. 287-296, Mar. 1966.

2 GRAU, C. R.; WILSON, B. W. Avian oogenesis and yolk deposition. Experientia, v. 20, n. 1, p. 26, Jan. 1964.

3 SKOOG, D. A.; HOLLER, F. J.; NIEMAN, T. A. Princípios de análise instrumental. 5. ed. Porto Alegre: Artmed, 2002, 836 p.

4 CHEW, B. P.; PARK, J. S. Carotenoid action on the immune response. J. Nutr., v. 134, n. 1, p. 257S261S, Jan. 2004.

5 DOTAS, D.; ZAMANIDIS, S.; BALIOS, J. Effect of dried tomato pulp on the performance and egg traits of laying hens. Br. Poult. Sci., v. 40, n. 5, p. 695-697, Dec. 1999.

6 FONTES para enriquecer alimentos com Luteína. Disponível em: < http://www.usda.gov/cnpp/ foodsupp.pdf $>$. Acesso em: 12 mar. 2004.

7 KRINSKY, N. I.; LANDRUM, J. L.; BONE, R. A. Biologic mechanisms of the protective role lutein and zeaxanthin in the eye. Annu. Rev. Nutr., v. 23, n. 7, p. 171-201, 2003.

8 MARES-PERLMAN, J. A.; KLEIN, R. Diet and agerelated macular degeneration, p. 285-300. In: TAYLOR, A. (Ed.). Nutritional and environmental influences on the eye. New York: CRC Press, 1999. p. 285-300.

9 SUAREZ, D. Incorporation of lycopene in egg yolk.
Poult. Sci., v. 48, n. 2, p. 733-735, 1969.

10 SURAI, A. P. et al. Effect of canthaxanthin content of the maternal diet on the antioxidant system of the developing chick. Br. Poult. Sci., v. 44, n. 4, p. 612619, Sep. 2003.

11 FRANCESCH, A. et al. Genetic parameters for egg number, egg weight, and eggshell color in three Catalan poultry breeds. Poult. Sci., v. 76, n. 12, p. 1627-1631, Dec. 1997.

12 STRADI, R.; PINI, E.; CELENTANO, G. Carotenoids in bird plumage: the complement of red pigments in the plumage of wild and captive bullfinch (Pyrrhula pyrrhula). Comp. Biochem. Physiol. B. Biochem. Mol. Biol., v. 128, n. 3, p. 529-535, 2001.

13 SOMMERBURG, O. et al. Fruits and vegetables that are sources for lutein and zeaxanthin: the macular pigment in human eyes. Br. J. Ophthalmol., v. 82, n. 8, p. 907-910, 1998.

14 LEESON, E.; SUMMERS, J. D. Scott's nutrition of the chicken. 4 th ed. Guelp: University Books, 2001. p. 199.

15 BEN-AMOTZ, A.; EDELSTEIN, S.; AVRON, A. Use of the b-carotene rich alga Dunaliella bardawil as a source of retinol. Br. Poult. Sci., v. 27, p. 613-619, 1986.

16 HUDON, J. Biotechnological applications of research on animal pigmentation. Biotechnol. Adv., v. 12, n. 1, p. 49-69, 1994

17 BELL, J. G. et. al. Flesh lipid and 5-carotenoid composition of scottish farmed atlantic salmon (Salmo salar). J. Agric. Food. Chem., v. 46, n. 1, p. 119-127, Jan. 1998. 
18 HERBER-MCNEILL, S. M.; VAN ELSWYK, M. E. Dietary marine algae maintains egg consumer acceptability while enhancing yolk color. Poult. Sci., v. 77, p. 493-496, 1998.

19 SILVA, J. H. V.; ALBINO, L. F. T.; GODÓI, M. J. S. Efeito do extrato de urucum na pigmentação da gema dos ovos. Rev. Bra. Zoot., v. 29, n. 5, p. 1435-1439, 2000.

20 PEDROSA, N. Generalidades sobre o urucum, 1997. Disponível em: <http://www.firace.com.br/ urucum.html >. Acesso em: 12 dez. 2003.

21. SCHRENK, W. G.; CHAPIN, D. S.; CONRAD, R. M. Ind. Eng. Chem., Anal. Ed. 16, 623:634,1964 apud MARUSICH, W. L.; BAUERNFEIND, J. C. Oxycarotenoids in poultry feeds. In: BAUERNFEIND, J. C. (Ed.). Carotenoids as colorants and vitamin A precursors: technological and nutritional applications. New York: Academic Press, 1981. p. 327.

22 MARUSICH, W. L.; BAUERNFEIND, J. C. Oxycarotenoids in poultry feeds. In: BAUERNFEIND, J. C. (Ed.). Carotenoids as colorants and vitamin A precursors: technological and nutritional applications. New York: Academic Press, 1981. p. 319-462.

23 NONES, K.; LIMA, G. J. M.; ZANOTTO, D. L. Metologia para determinação da intensidade de coloração em grãos de milho. Comunicado Técnico, Embrapa Suínos e Aves, n. 260, p. 1-2, nov. 2000.

24 JIANG, Y. H.; MCGEACHIN, R. B.; BAILEY, C. A. Alfa-tocopherol, beta-carotene, and retinol enrichment of chickens eggs. Poult. Sci., v. 73, p. 1137-1143, 1994.

25 MADIEDO, G.; RICHTER, E. F.; SUNDE, M. L. A comparison between chemical determination for xanthophylls and yolk pigmentation scores for yellow corn, alfafa, algae, lake weed, and marigold petals. Poult. Sci., v. 43, p. 990-994, 1964.

26 BARTOV. I.; BORNSTEIN, S. Studies on egg yolk pigmentation. 2. Effect of ethoxyquin on xanthophyll utilization. Poult. Sci., v. 45, n. 2, p. 297-305, Mar. 1966.

27 BURDICK, D.; FLETCHER, D. L. Utilization of xanthophyll in dehydrated alfalfa and coastal Bermuda grass for pigmenting egg yolks. Poult. Sci., v. 62, p. I339, 1983

28 BARTOV, I.; BORNSTEIN, S. Studies on egg yolk pigmentation. 3. The effect of origin and storage conditions of yellow corn on the utilization of its xanthophyll. Poult. Sci., v. 46, n. 4, p. 796- 804, July 1967.

29 FLETCHER, D. L. et al. Strain differences in egg yolk pigmentation. Poult. Sci., v. 56, p. 349, 1977.

30 NRC. Nutrient requirements of poultry, 9. ed. Washington: National Academy of Sciences, 1994. $155 \mathrm{p}$.
31 VUILLEUMIER, J. P. The "Roche yolk colour fan" - na instrument for measuring yolk colour. Poult. Sci. v. 48, p. 767-779, 1969.

32 AOAC. Official methods of analysis. Eggs and egg products. 5. ed., 1990.

$33 \mathrm{HAUGH}, \mathrm{R}$. R. The Haugh unit for measuring egg quality. The U.S. Egg \& Poult. Mag., v. 43, n. 9, p. 552555, 572-573, 1937.

34 SAS. SAS ${ }^{\circledR}$ User's guide: statistics. Cary: SAS Institute, 1994.

35 CAFÉ, M. B. et al. Milheto-grão (Pennisetum glaucum (L.) R. Br.) como substituto do milho em rações para poedeiras comerciais. Arq. Bras. Med. Vet. Zootec., v. 51, n. 2, p. 171-176, 1999.

36 HERBER, S. M.; VAN ELSWYK, M. E. Dietary marine algae promotes efficients deposition of n-3 fatty acids for the productions of enriched shell eggs. Poult. Sci., v. 75 , p. 1501-1507, 1996.

37 OLIVEIRA, B. L. Pontos críticos no manejo de poedeiras. In: CONFERÊNCIA APINCO DE CIÊNCIA E TECNOLOGIA AVÍCOLA, 1992, Santos. Anais... São Paulo: FACTA, 1992. p. 137-144.

38 SCHOFIELD, O.; EVENS,T. J.; MILLIE, D. F. Photosystem i quantum yields and xanthophyll-cycle pigments of the macroalga Sargassum natans (phaeophyceae): responses under natural sunlight. J. Phycol., v. 34, n. 1, p. 104, Fev. 1998.

39 PEREZ-VENDRELL, A. M. et al. Influence of source and ratio of xanthophyll pigments on broiler chicken pigmentation and performance. Poult. Sci., v. 80, n. 3, p. 320-326, Mar. 2001.

40 U.S. FOOD AND DRUG ADMINISTRATION. Center for Veterinary Medicine. Guidelines for evaluation of effectiveness of new animal drugs for use in poultry feeds for pigmentation. Guideline $n$. 37, 1984. Disponível em: <http://www.fda.gov/cvm/ guidance/published.htm > . Acesso em: 10 de abr. 2004.

41 FLETCHER, D. L. An evaluation of the A.O.A.C. method of yolk color analysis. Poult. Sci., v. 58, p. I057, 1979.

42 FLETCHER, D. L. A method for estimating relative saponification of xanthophyll containing feedstuffs. Poult. Sci., v. 65, p. 43, 1986. Supplement.

43 MAINGUY, P.; ROUQUES, A. The yellow of the egg. I. General study of the color of the egg yolk. Aliment. Vie., v. 53, n. 4, p. 83-116, 1965.

44 INSTITUTO ADOLFO LUTZ. Normas analíticas do Instituto Adolfo Lutz: métodos químicos e físicos para análise de alimentos. Vitaminas e Corantes. 3. ed. São Paulo: Instituto Adolfo Lutz, 1985. p. 378-416. 